\title{
4.3 A SIMPLE MODEL FOR VERTICAL TRANSPORT OF REACTIVE SPECIES IN THE CONVECTIVE ATMOSPHERIC BOUNDARY LAYER
}

\author{
Leif Kristensen ${ }^{1 *}$ Donald H. Lenschow ${ }^{2}$, David Gurarie ${ }^{3}$, and Niels Otto Jensen ${ }^{1}$ \\ ${ }^{1}$ Ris $\varnothing$ DTU National Laboratory for Sustainable Energy, Roskilde, Denmark \\ ${ }^{2}$ National Center for Atmospheric Research, Boulder, CO \\ ${ }^{3}$ Department of Mathematics, Case Western Reserve University, Cleveland, $\mathrm{OH}$
}

\section{INTRODUCTION}

The convective planetary boundary layer (CBL) is a melting pot of chemical species-some are emitted by surface sources, others are entrained from the overlying free atmosphere, and still others are created by reactions with other species or through photochemistry. This rich soup of reactive species is stirred by turbulence; the degree of commingling determined by the ratio of the time scale of the turbulence mixing to the time scale of chemical reaction, which is known as the Damköhler number. The turbulence mixing time scale, in turn, is controlled by the dominant eddy size and the turbulence intensity, which are functions of normalized CBL depth and the surface buoyancy flux. All in all, this makes for a complicated set of interactions that need to be dealt with to determine the vertical structure of mean concentrations and turbulence statistics.

In order to deal with this complexity, and identify the individual roles that turbulence and chemical reactivity play, it is useful to simplify and parameterize the processes so that models can be constructed that can be easily modified so that the parameter space can be easily explored. With this in mind, we have developed a simple one-dimensional model, patterned after the surfacelayer models developed by Kristensen et al. (1997) for one first-order destruction process and Kristensen and Kirkegaard (2006) for one second-order destruction process, and extended it to the entire CBL, parameterizing the transport process throughout the CBL. We have applied it to a set of fast chemical reactants in the CBL that involves the odd nitrogen species $\mathrm{NO}$ and $\mathrm{NO}_{2}\left(=\mathrm{NO}_{x}\right)$, as well as $\mathrm{O}_{3}$. As we shall see this involves one firstorder process and one second-order process.

Our model is similar to that of Verver et al. (1997) and Verver et al. (2000) who carried out similar studies using a second-order closure model to calculate profiles of mean and turbulence statistics. However, instead of solv-

\footnotetext{
${ }^{*}$ Corresponding address: Leif Kristensen, Risø DTU National Laboratory for Sustainable Energy, Roskilde, Denmark. E-mail: leif.kristensen@tele2.dk
}

ing the set of nine or more differential equations by finite difference techniques at a specified set of levels throughout the CBL as they did, we first reduce the set of equations to two equations for $\mathrm{NO}_{2}$ flux and concentration by using mass conservation of the radicals involved in the $\mathrm{NO}-\mathrm{NO}_{2}-\mathrm{O}_{3}$ triad. We then obtain a numerical solution of these differential equations, and subsequently the entire set of concentration and flux profiles are easily obtained by substituting this solution into the equations for $\mathrm{NO}$ and $\mathrm{O}_{3}$ fluxes and concentrations. The advantages of this approach are: 1) we can easily specify whatever vertical resolution we wish over any interval of the CBL, including using very fine resolution to resolve detailed structure in the surface layer; 2) solving the equations requires little computer time and capacity; and 3) it is easy to make changes in the transport and chemical parameterizations, and to quickly evaluate their impact on the resulting concentration and flux profiles.

The eventual goal is to extend the model to include volatile organic compounds (VOCs) - particularly those emitted by vegetation (e.g. isoprene) - that react on time scales comparable to CBL turbulent mixing times, to provide a simple tool for rapid estimation of surface emission rates, and possibly reaction rates, given measurements of VOC fluxes or profiles (or both) at one or more levels in the CBL. This approach again was anticipated by Verver et al. (2000), who also used their modeling technique to study the flux-gradient relationship for isoprene in the planetary boundary layer.

In the following we first derive the equations for the mean concentration and flux of a conserved species. In this way we obtain a fundamental scheme to be applied when the chemistry is included. At this stage the equations can be simplified by making them dimensionless (section 3). A number of turbulence statistics profiles, such as variances and heat flux, are prescribed and discussed in section 4. In section 5, the $\mathrm{NO}_{x}-\mathrm{O}_{3}$ triad is discussed as a simple example of the interaction between non-conserved species. With the tools to solve for concentrations and fluxes now in place, we outline in section 6 the procedure we have chosen for solving the equa- 
tions. Finally, a sample case is presented in section 7.

\section{CONSERVED SPECIES}

We consider a horizontally homogeneous and stationary flow, and a Cartesian coordinate system where the unit vector $\mathbf{i}$ is aligned with the mean wind, $\mathbf{k}$ vertical, and $\mathbf{j}=\mathbf{k} \times \mathbf{i}$. The position is characterized by

$$
\mathbf{x}=x \mathbf{i}+y \mathbf{j}+z \mathbf{k}
$$

and the instantaneous velocity vector by

$$
\widetilde{\mathbf{u}}(\mathbf{x}, t)=\widetilde{u}(\mathbf{x}, t) \mathbf{i}+\widetilde{v}(\mathbf{x}, t) \mathbf{j}+\widetilde{w}(\mathbf{x}, t) \mathbf{k}
$$

In the CBL, the mean wind is assumed constant in magnitude and direction, and the velocity field is decomposed into a mean and fluctuation:

$$
\widetilde{\mathbf{u}}(\mathbf{x}, t)=U \mathbf{i}+u(\mathbf{x}, t) \mathbf{i}+v(\mathbf{x}, t) \mathbf{j}+w(\mathbf{x}, t) \mathbf{k}
$$

where

$$
U=|\langle\widetilde{\mathbf{u}}(\mathbf{x}, t)\rangle|
$$

is the space- and time-independent mean wind speed.

The potential temperature $\widetilde{\Theta}^{*}$ and the conserved scalar concentration $\widetilde{s}$ are similarly decomposed into means and fluctuations

$$
\widetilde{\Theta}=\Theta(z, t)+\vartheta(\mathbf{x}, t)
$$

where we allow for a temporal change of the mean potential temperature due to the temperature flux divergence resulting from the difference between the surface heat flux and entrainment of heat from the free troposphere into the CBL, and

$$
\widetilde{s}=S(z)+s(\mathbf{x}, t),
$$

where we neglect time change of the mean concentration $S$ since we are seeking steady-state solutions for mean concentration and flux.

These two quantities follow the equations

$$
\frac{D \widetilde{\Theta}}{D t}=v_{\vartheta} \nabla^{2} \widetilde{\Theta}
$$

and

$$
\frac{D \widetilde{s}}{D t}=v_{s} \nabla^{2} \widetilde{s}
$$

where $v_{\vartheta}$ and $v_{s}$ are the molecular diffusivities, and

$$
\frac{D}{D t} \equiv \frac{\partial}{\partial t}+\widetilde{\mathbf{u}} \cdot \nabla=\frac{\partial}{\partial t}+U \frac{\partial}{\partial x}+\mathbf{u} \cdot \nabla
$$

\footnotetext{
*We use the term potential temperature, but in the convective boundary layer we really mean the virtual potential temperature, where the effect of humidity on air density has been included.
}

is the Lagrangian time derivative. Now (7) and (8) become

$$
\begin{array}{r}
\left\{\frac{\partial}{\partial t}+U \frac{\partial}{\partial x}\right\} \vartheta+w \frac{\partial \Theta}{\partial z}+\nabla \cdot(\mathbf{u} \vartheta)+\frac{\partial \Theta}{\partial t}= \\
v_{\vartheta} \frac{\partial^{2} \Theta}{\partial z^{2}}+v_{\vartheta} \nabla^{2} \vartheta
\end{array}
$$

and

$$
\left\{\frac{\partial}{\partial t}+U \frac{\partial}{\partial x}\right\} s+w \frac{\partial S}{\partial z}+\nabla \cdot(\mathbf{u} s)=v_{s} \frac{\partial^{2} S}{\partial z^{2}}+v_{s} \nabla^{2} s .
$$

Taking the average of each of (10) and of (11), we get

$$
\frac{\partial\langle w \vartheta\rangle}{\partial z}=-\frac{\partial \Theta}{\partial t}+v_{\vartheta} \frac{\partial^{2} \Theta}{\partial z^{2}}
$$

and

$$
\frac{\partial\langle w s\rangle}{\partial z}=v_{s} \frac{\partial^{2} S}{\partial z^{2}}
$$

Integrating (12) and (13) leads to

$$
\langle w \vartheta\rangle=\langle w \vartheta\rangle_{\circ}-\int_{0}^{z} \frac{\partial \Theta\left(z^{\prime}, t\right)}{\partial t} \mathrm{~d} z^{\prime}+\underbrace{\left.v_{\vartheta} \frac{\partial \Theta}{\partial z}\right|_{z}}_{\simeq 0}
$$

and

$$
\langle w s\rangle=\langle w s\rangle_{\circ}+\underbrace{\left.v_{s} \frac{\partial S}{\partial z}\right|_{z}}_{\simeq 0}
$$

where the molecular diffusion terms can be neglected. In the well-mixed CBL the variation of the potential temperature with height can be ignored, i.e.,

$$
\frac{\partial \Theta}{\partial z}=0
$$

Since $\Theta$ in the CBL is an increasing function of time, the heat flux is, according to (14), a decreasing, linear function of height. As (15) shows, the flux $\langle w s\rangle$ is constant with height.

By subtracting these mean equations from the corresponding full equations (10) and (11) we obtain equations for the fluctuating quantities $\vartheta$ and $s$,

$$
\left\{\frac{\partial}{\partial t}+U \frac{\partial}{\partial x}\right\} \vartheta+\nabla \cdot(\mathbf{u} \vartheta)-\frac{\partial}{\partial z}\langle w \vartheta\rangle=v_{\vartheta} \nabla^{2} \vartheta
$$

and

$$
\left\{\frac{\partial}{\partial t}+U \frac{\partial}{\partial x}\right\} s+w \frac{\partial S}{\partial z}+\nabla \cdot(\mathbf{u} s)-\frac{\partial}{\partial z}\langle w s\rangle=v_{s} \nabla^{2} s .
$$


We also need the equation for the fluctuating vertical velocity $w$. This can be obtained from the conservation equation for momentum-the Navier-Stokes equation. In a similar way as how we used (10) and (11) to obtain (17) and (18), we get (e.g. Busch (1973)):

$$
\begin{aligned}
\left\{\frac{\partial}{\partial t}+U \frac{\partial}{\partial x}\right\} w & +\nabla \cdot(\mathbf{u} w)-\frac{\partial\left\langle w^{2}\right\rangle}{\partial z}= \\
& -\frac{1}{\rho} \frac{\partial p}{\partial z}+\frac{g}{T} \vartheta+v \nabla^{2} w
\end{aligned}
$$

where $\rho$ is the air density, $p$ the fluctuating part of the static pressure, $g$ the acceleration of gravity, $T$ the average temperature of the boundary layer, and $v$ the kinematic viscosity.

Combining (18) and (19) we obtain by averaging

$$
\begin{aligned}
& \left\langle w^{2}\right\rangle \frac{\partial S}{\partial z}+\frac{\partial}{\partial z}\left\langle w^{2} s\right\rangle= \\
& -\frac{1}{\rho}\left\langle s \frac{\partial p}{\partial z}\right\rangle+\frac{g}{T}\langle\vartheta s\rangle+v_{s}\left\langle w \nabla^{2} s\right\rangle+v\left\langle s \nabla^{2} w\right\rangle .
\end{aligned}
$$

Following André et al. (1976), Moeng and Wyngaard (1989), and Verver et al. (1997) we use the parameterization

$$
\frac{1}{\rho}\left\langle s \frac{\partial p}{\partial z}\right\rangle=\frac{\langle w s\rangle}{\tau_{1}}+b_{1} \frac{g}{T}\langle\vartheta s\rangle
$$

where $b_{1} \simeq 0.4$ is a dimensionless constant and $\tau_{1}=$ $\tau_{1}(z)$ "the return to isotropy" time scale.

The sum of the last two terms in (20) can be combined as

$$
v_{s}\left\langle w \nabla^{2} s\right\rangle+v\left\langle s \nabla^{2} w\right\rangle=-\left(v_{s}+v\right)\langle\nabla w \cdot \nabla s\rangle
$$

For small-scale, isotropic turbulence this sum is zero, as pointed out by Wyngaard (1982) (p. 82). Neglecting third-order terms, (20) can be written as

$$
\left\langle w^{2}\right\rangle\left\{\frac{\partial S}{\partial z}+\frac{\langle w s\rangle}{\left\langle w^{2}\right\rangle \tau_{1}}\right\}=\left(1-b_{1}\right) \frac{g}{T}\langle\vartheta s\rangle .
$$

To close the problem we derive an equation for the covariance $\langle\vartheta s\rangle$ by combining (17) and (18):

$$
\langle w \vartheta\rangle \frac{\partial S}{\partial z}+\frac{\partial}{\partial z}\langle w \vartheta s\rangle=-\left(v_{\vartheta}+v_{s}\right)\langle\nabla \vartheta \cdot \nabla s\rangle .
$$

Following Verver et al. (1997) the right-hand side of (24) can be expressed in terms of a height dependent dissipation time scale $\tau_{4}=\tau_{4}(z)$ :

$$
\left(v_{\vartheta}+v_{s}\right)\langle\nabla \vartheta \cdot \nabla s\rangle=\frac{\langle\vartheta s\rangle}{\tau_{4}} .
$$

Finally, (24) can be written

$$
\langle w \vartheta\rangle \frac{\partial S}{\partial z}+\frac{\langle\vartheta s\rangle}{\tau_{4}(z)}=0
$$

where third-order terms have been neglected. With the prescribed parameter functions $\left\langle w^{2}\right\rangle,\langle w \vartheta\rangle, \tau_{4}(z), \tau_{1}(z)$, and the constant $\left(1-b_{1}\right) g / T,(15),(23)$ and (26) can be solved for the gradient $\partial S / \partial z$ and the covariance $\langle\vartheta s\rangle$. As (15) shows, we have already one integral constraint, namely the constant flux

$$
\langle w s\rangle=\langle w s\rangle_{\circ} .
$$

The two equations to be solved, therefore, are

$$
\left\langle w^{2}\right\rangle\left\{\frac{\partial S}{\partial z}+\frac{\langle w s\rangle_{\circ}}{\left\langle w^{2}\right\rangle \tau_{1}}\right\}=\left(1-b_{1}\right) \frac{g}{T}\langle\vartheta s\rangle .
$$

and (26).

\section{DIMENSIONLESS FORMULATION}

For computational purposes it is advantageous to recast the equations in a dimensionless form. We have three quantities which characterize the equations: the height of the CBL $h$, the surface temperature flux $\langle w \vartheta\rangle_{\circ}$, and the buoyancy parameter $g / T$. They are all considered constant in space and quasi-constant in time. The buoyancy parameter can safely be considered constant in time, but the quantities $\langle w \vartheta\rangle_{\circ}$ and $h$ are of course varying with time in the convective boundary layer. However, their rate of change is considered so small that here we may take them as constants. We define the convective velocity scale ${ }^{\dagger}$ by

$$
w_{*}=\left(\left(1-b_{1}\right) \frac{g}{T}\langle w \vartheta\rangle_{\circ} h\right)^{1 / 3},
$$

the dimensionless quantities by

$$
\left\{\begin{array}{l}
\zeta \\
f \\
\gamma
\end{array}\right\}=\left\{\begin{array}{r}
z / h \\
\langle w s\rangle / w_{*} \\
\langle\vartheta s\rangle / T_{*}
\end{array}\right\}
$$

and the parameters to be specified by

$$
\left\{\begin{array}{c}
\theta_{1} \\
\theta_{4} \\
\omega^{2} \\
\varphi_{T}
\end{array}\right\}=\left\{\begin{array}{r}
w_{*} \tau_{1}(z) / h \\
w_{*} \tau_{4}(z) / h \\
\left\langle w^{2}\right\rangle / w_{*}^{2} \\
\langle w \vartheta\rangle /\left(w_{*} T_{*}\right)
\end{array}\right\},
$$

where

$$
T_{*}=\frac{\langle w \vartheta\rangle_{\circ}}{w_{*}} .
$$

The three equations (27), (28), and (26) now take the form

$$
\begin{gathered}
f=f_{\circ}, \\
\omega^{2} \frac{\partial S}{\partial \zeta}-\gamma=-\frac{f_{\circ}}{\theta_{1}},
\end{gathered}
$$

\footnotetext{
${ }^{\dagger}$ For convenience we have included the factor $\left(1-b_{1}\right) \simeq 0.6$ in the definition in the convective velocity scale.
} 
and

$$
\varphi_{T} \frac{\partial S}{\partial \zeta}+\frac{\gamma}{\theta_{4}}=0
$$

Specifying $f_{\circ}$, we may solve (34) and (35) for $\partial S / \partial \zeta$ and $\gamma$ to obtain

$$
\frac{\frac{\partial S}{\partial \zeta}}{f_{\circ}}=-\frac{1}{\theta_{1}\left(\omega^{2}+\theta_{4} \varphi_{T}\right)}
$$

and

$$
\frac{\gamma}{f_{\circ}}=\frac{\theta_{4} \varphi_{T}}{\theta_{1}\left(\omega^{2}+\theta_{4} \varphi_{T}\right)} .
$$

The right-hand sides of these two equations are considered known and we see that (36) is a (dimensionless) relation between the flux and the gradient of the corresponding scalar; that is, it defines the dimensionless turbulent diffusivity

$$
\chi(\zeta) \equiv \frac{K}{w_{*} h}=\theta_{1}\left(\omega^{2}+\theta_{4} \varphi_{T}\right),
$$

where $K(z)$ is the turbulent diffusivity for passive scalars.

\section{PARAMETERIZING THE STRUCTURE OF THE CONVECTIVE BOUNDARY LAYER}

The equations require profiles of the variances of the vertical velocity $\left\langle w^{2}\right\rangle$ and the potential temperature $\left\langle\vartheta^{2}\right\rangle$, and of the temperature flux $\langle w \vartheta\rangle$, in addition to the time constants $\tau_{1}$ and $\tau_{4}$.

We use the formulation by Lenschow et al. (1980) for $\left\langle w^{2}\right\rangle$

$$
\omega^{2}=\frac{\left\langle w^{2}\right\rangle}{w_{*}^{2}}=1.8\left(\frac{z}{h}\right)^{2 / 3}\left(1-0.8 \frac{z}{h}\right)^{2} .
$$

The variance profile used here converges to the free convection limit in the surface layer; that is, for simplicity we have neglected the contribution of shear production. This means that for small $z / h,\left\langle w^{2}\right\rangle / w_{*}^{2} \propto(z / h)^{2 / 3}$.

The heat flux $\langle w \vartheta\rangle$ decreases linearly from a positive value $\langle w \vartheta\rangle_{\circ}$ at the ground to a negative value at the top $z=h$ of the boundary layer due to entrainment from aloft. We apply the simple analysis by Tennekes (1973) and assume the expression

$$
\varphi_{T}=\frac{\langle w \vartheta\rangle}{\langle w \vartheta\rangle_{\circ}}=\frac{\langle w \vartheta\rangle}{w_{*} T_{*}}=1-1.2 \frac{z}{h}
$$

\footnotetext{
\$The factor 1.2 implies that the entrainment heat flux at the CBL top is 20\% (downwards) of the surface heat flux (upwards). It is not a fixed fraction, but only a typical value; it varies with the turbulence intensity in terms of e.g. $w_{*}$, the wind shear, and the temperature stratification in the overlying free troposphere.
}

The time constants entering (21) and (25) are set to (Verver et al., 1997)

$$
\tau_{i}=\frac{10}{a_{i}} \frac{\kappa z(1-z / h)}{\left\langle w^{2}\right\rangle^{1 / 2}}, \quad i=1,4,
$$

where $a_{i}$ are dimensionless constants and $\kappa=0.4$ the von Kármán constant. Inserting (39) we get

$$
\theta_{i}=\frac{h \tau_{i}}{w_{*}}=\frac{3}{a_{i}} \frac{\left(\frac{z}{h}\right)^{2 / 3}\left(1-\frac{z}{h}\right)}{1-0.8 \frac{z}{h}}, \quad i=1,4 .
$$

Inserting (39), (40), and (42) into (37) and (38) we have determined the dimensionless temperature-scalar covariance $\gamma$ and the dimensionless turbulent diffusivity $\chi$, except for the two dimensionless constants $a_{1}$ and $a_{4}$. However, we have one constraint in a relation between these two constants, namely the functional form of $\chi(\zeta)$ at the bottom of the boundary layer. We assume in accordance with Wyngaard and Brost (1984), and Holtslag and Moeng (1991) that near the surface the diffusivity approaches

$$
\chi(\zeta)=\zeta^{4 / 3}
$$

This is consistent with our expression for $\chi(\zeta)$ that includes the parameter functions (39), (40), and (42) if the identity

$$
\frac{3}{a_{1}}\left(1.8+\frac{3}{a_{4}}\right)=1
$$

is true. Verver et al. (1997) use $\left(a_{1}, a_{4}\right)=(4.85,2.5)$. These values do not fulfill (44). We find, with our choice of dimensionless parameters in (39), (40), and (42), that for (44) to be true, the only two possibilities are $\left(a_{1}, a_{4}\right)=(9,2.5)$ and $\left(a_{1}, a_{4}\right)=(4.85,-16.4)$. Since the second possibility is unphysical we use the first possibility in the following. The profiles of the dimensionless diffusivity and the dimensionless temperature-scalar covariance are shown in Figs. 1 and 2.

\section{NON-CONSERVED SPECIES}

When we have gases which interact, the rate equation (8) must be generalized to account for the extra sources and sinks inside the boundary layer. Instead of (8) we write for $N$ mutually interacting species

$$
\frac{D \widetilde{s_{i}}}{D t}=\widetilde{\mathcal{R}}_{i}+v_{s} \nabla^{2} \widetilde{s_{i}}, \quad i=1,2, \ldots, N
$$

where $\widetilde{\mathcal{R}}_{i}$ represents the rate of concentration increase due to the interaction with all the other species and where the kinematic diffusivity $v_{s}$ is assumed the same for all the species. As in the case of conserved species, we decompose $\widetilde{s_{i}}$ into a mean and fluctuations:

$$
\widetilde{s_{i}}(x, y, z, t)=S_{i}(z, t)+s_{i}(x, y, z, t), \quad i=1,2, \ldots, N .
$$




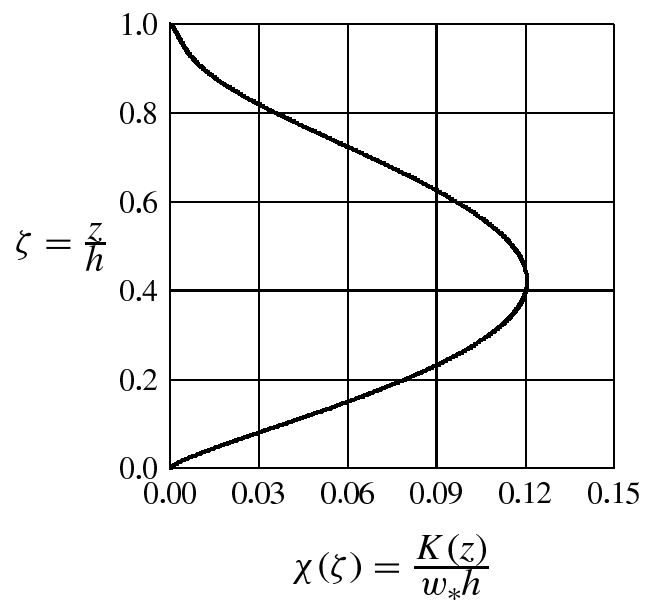

Figure 1: The dimensionless diffusivity profile $K /\left(w_{*} h\right)$.

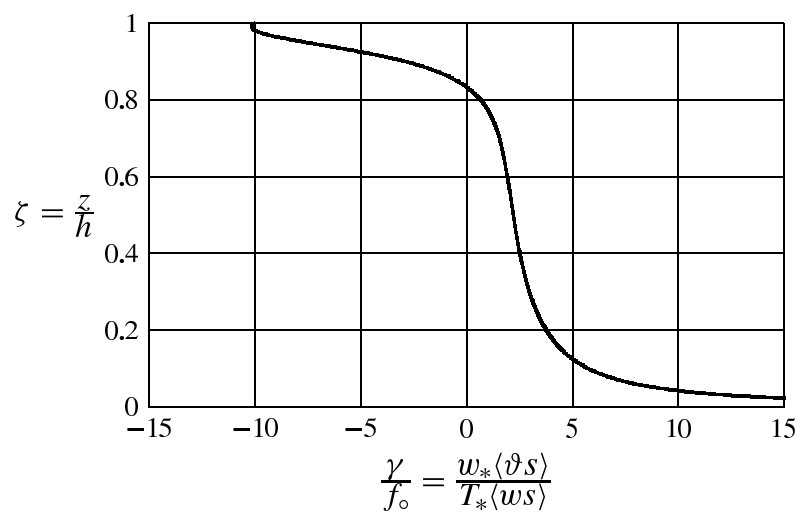

Figure 2: Profile of the dimensionless ratio of the temperature-scalar covariance and the constant scalar flux (37).

In a similar way we decompose

$$
\widetilde{\mathcal{R}}_{i}(x, y, z, t)=\mathcal{R}_{\mathrm{i}}(z, t)+r_{i}(x, y, z, t), \quad i=1,2, \ldots, N,
$$

where

$$
\mathcal{R}_{i}=\left\langle\widetilde{R}_{i}\right\rangle .
$$

The equations for the mean $S_{i}$ and fluctuations $\widetilde{s_{i}}$ in (45) become the following generalizations of (13) and (18)

$$
\frac{\partial S_{i}}{\partial t}+\frac{\partial\langle w s\rangle}{\partial z}=\mathcal{R}_{i}+v_{s} \frac{\partial^{2} S}{\partial z^{2}}
$$

where we have kept the prognostic term, and

$$
\begin{gathered}
\left\{\frac{\partial}{\partial t}+U \frac{\partial}{\partial x}\right\} s_{i}+w \frac{\partial S_{i}}{\partial z}+\nabla \cdot\left(\mathbf{u} s_{i}\right)-\frac{\partial}{\partial z}\left\langle w s_{i}\right\rangle= \\
r_{i}+v_{s} \nabla^{2} s_{i} .
\end{gathered}
$$

Combining this equation with (17) and (19) we obtain the three prognostic equations

$$
\begin{gathered}
\frac{\partial}{\partial t}\left\langle w s_{i}\right\rangle+\left\langle w^{2}\right\rangle \frac{\partial S_{i}}{\partial z}+\frac{\partial}{\partial z}\left\langle w^{2} s_{i}\right\rangle= \\
-\frac{1}{\rho}\left\langle s_{i} \frac{\partial p}{\partial z}\right\rangle+\frac{g}{T}\left\langle\vartheta s_{i}\right\rangle+\left\langle w r_{i}\right\rangle \\
-\underbrace{\left(v+v_{s}\right)\left\langle\nabla w \cdot \nabla s_{i}\right\rangle}_{\simeq 0 \text { Wyngaard (1982) }}, \\
\frac{\partial}{\partial t}\left\langle\vartheta s_{i}\right\rangle+\langle w \vartheta\rangle \frac{\partial S_{i}}{\partial z}+\frac{\partial}{\partial z}\left\langle w \vartheta s_{i}\right\rangle= \\
\left\langle\vartheta s_{i}\right\rangle+\left\langle r_{i} \vartheta\right\rangle-\left(v_{\vartheta}+v_{s}\right)\left\langle\nabla \vartheta \cdot \nabla s_{i}\right\rangle,
\end{gathered}
$$

and

$$
\begin{gathered}
\frac{\partial}{\partial t}\left\langle s_{i} s_{j}\right\rangle+\left\langle w s_{i}\right\rangle \frac{\partial S_{j}}{\partial z}+\left\langle w s_{j}\right\rangle \frac{\partial S_{i}}{\partial z}+\frac{\partial}{\partial z}\left\langle w s_{i} s_{j}\right\rangle= \\
\left\langle r_{i} r_{j}\right\rangle-2 v_{s}\left\langle\nabla s_{i} \cdot \nabla s_{j}\right\rangle .
\end{gathered}
$$

These three equations (51), (52), and (53), together with (49) constitute our general set of equations for $N$ interactive gas species in the CBL. To proceed we must specify their interactions in terms of $\widetilde{R}_{i}$ for $i=$ $1,2, \cdots, N$. Below this is illustrated by the simple case with $N=3$.

The $\mathrm{NO}_{\mathrm{x}}-\mathrm{O}_{3}$ triad reactions (Leighton, 1961) in a sunlit $\mathrm{CBL}$ are

$$
\begin{aligned}
\mathrm{O}\left({ }^{3} \mathrm{P}\right)+\mathrm{O}_{2} & \rightarrow \mathrm{O}_{3} \\
\mathrm{NO}+\mathrm{O}_{3} & \rightarrow \mathrm{NO}_{2}+\mathrm{O}_{2} \\
\mathrm{NO}_{2}+h \mathrm{~h} & \rightarrow \mathrm{NO}+\mathrm{O}\left({ }^{3} \mathrm{P}\right)
\end{aligned}
$$

Here $h v$ represents one quantum of ultraviolet light while ${ }^{3} \mathrm{P}$ indicates that the atomic oxygen is in its ground state.

If there is a large supply of $\mathrm{O}_{3}$, as is often the case in an urban environment, the process (55) will be the main mechanism of NO removal. In rural and remote areas NO will often be destroyed by other peroxy radicals (Ridley et al., 1992). However, following Lenschow and Delany (1987), we assume that a first simple approach to a calculation of the mean concentrations and fluxes of $\mathrm{O}, \mathrm{O}_{3}$, $\mathrm{NO}$, and $\mathrm{NO}_{2}$ can be based on the reactions (54), (55), and (56). The corresponding dynamic equations governing these transformations, as well as the molecular diffusion and turbulent transport of the four species $\mathrm{O}, \mathrm{O}_{3}$, 
$\mathrm{NO}$, and $\mathrm{NO}_{2}$ are

$$
\begin{aligned}
\frac{D[\mathrm{O}]}{D t}= & -\frac{[\mathrm{O}]}{\tau_{\mathrm{O}}}+\frac{\left[\mathrm{NO}_{2}\right]}{\tau_{\mathrm{NO}_{2}}}+v_{\mathrm{O}} \frac{\partial^{2}[\mathrm{O}]}{\partial x_{i} \partial x_{i}} \\
\frac{D\left[\mathrm{O}_{3}\right]}{D t}= & -k\left[\mathrm{O}_{3}\right][\mathrm{NO}]+\frac{[\mathrm{O}]}{\tau_{\mathrm{O}}}+v_{\mathrm{O}_{3}} \frac{\partial^{2}\left[\mathrm{O}_{3}\right]}{\partial x_{i} \partial x_{i}} \\
\frac{D[\mathrm{NO}]}{D t}= & -k\left[\mathrm{O}_{3}\right][\mathrm{NO}]+\frac{\left[\mathrm{NO}_{2}\right]}{\tau_{\mathrm{NO}_{2}}} \\
& +v_{\mathrm{NO}} \frac{\partial^{2}[\mathrm{NO}]}{\partial x_{i} \partial x_{i}} \\
\frac{D\left[\mathrm{NO}_{2}\right]}{D t}= & -\frac{\left[\mathrm{NO}_{2}\right]}{\tau_{\mathrm{NO}_{2}}}+k\left[\mathrm{O}_{3}\right][\mathrm{NO}] \\
& +v_{\mathrm{NO}_{2}} \frac{\partial^{2}\left[\mathrm{NO}_{2}\right]}{\partial x_{i} \partial x_{i}}
\end{aligned}
$$

The quantities $\tau_{\mathrm{O}}$ and $\tau_{\mathrm{NO}_{2}}$ are the mean life-times for the first-order processes, $k$ the reaction constant for the second-order process and $v_{\mathrm{O}}, v_{\mathrm{O}_{3}}, v_{\mathrm{NO}}$, and $v_{\mathrm{NO}_{2}}$ the molecular diffusivities.

In (57) the first two terms are large compared to the other terms so there is an effective balance between destruction of $\mathrm{O}\left({ }^{3} \mathrm{P}\right)$ and creation of $\mathrm{NO}_{2}$. For this reason $\mathrm{O}\left({ }^{3} \mathrm{P}\right)$ need not be further considered here, and we consider only the one first-order time constant $\tau=\tau_{\mathrm{NO}_{2}}$.

For convenience we introduce the dimensionless concentrations

$$
\left\{\begin{array}{c}
\widetilde{s}_{1} \\
\widetilde{s}_{2} \\
\widetilde{s}_{3}
\end{array}\right\}=k \tau \times\left\{\begin{array}{c}
{\left[\mathrm{O}_{3}\right]} \\
{[\mathrm{NO}]} \\
{\left[\mathrm{NO}_{2}\right]}
\end{array}\right\},
$$

where all three quantities are functions of Cartesian position $(x, y, z)$ and time $t$. The equation (8) for conserved species must now be replaced by

$$
\frac{D \widetilde{s_{i}}}{D t}=\left(i^{2}-3 i+1\right) \widetilde{\mathcal{R}}+v_{s} \nabla^{2} \widetilde{s_{i}}, \quad i=1,2,3,
$$

where the molecular diffusivity is assumed the same $v_{s}$ for all the scalars, and where

$$
\widetilde{\mathcal{R}}=\frac{1}{\tau}\left(\widetilde{s}_{1} \widetilde{s}_{2}-\widetilde{s}_{3}\right)
$$

describes the reaction rates. Defining the sum concentrations as

$$
\widetilde{c}_{i}=\widetilde{s}_{i}+\widetilde{s_{3}}, \quad i=1,2
$$

and neglecting molecular diffusion in (53), we get the two conservation equations

$$
\frac{D \widetilde{c_{i}}}{D t}=0, \quad i=1,2
$$

of the two sum-concentrations. These behave, in other words, like conserved species.

Similarly, we get for the sum-concentrations ${ }^{\S}$

$$
\widetilde{c}_{i}=C_{i}(z)+c_{i}(x, y, z, t), \quad i=1,2 .
$$

The rate equations (53) take the form

$$
\begin{aligned}
& \left\{\frac{\partial}{\partial t}+U \frac{\partial}{\partial x}\right\}\left(S_{i}+s_{i}\right)+w \frac{\partial S_{i}}{\partial z}+\nabla \cdot\left(\mathbf{u} s_{i}\right)= \\
& \left(i^{2}-3 i+1\right) \widetilde{R}+v_{s} \nabla^{2}\left(S_{i}+s_{i}\right), \quad i=1,2,3 .
\end{aligned}
$$

Taking the mean of this equation, we get

$$
\frac{\partial S_{i}}{\partial t}+\frac{\partial}{\partial z}\left\langle w s_{i}\right\rangle=\left(i^{2}-3 i+1\right)\langle\widetilde{\mathcal{R}}\rangle+\underbrace{v_{s} \frac{\partial^{2} S_{i}}{\partial z^{2}}}_{\simeq 0} .
$$

The equation for the fluctuating part $s_{i}$ is obtained from (67) and (68):

$$
\begin{gathered}
\left\{\frac{\partial}{\partial t}+U \frac{\partial}{\partial x}\right\} s_{i}+w \frac{\partial S_{i}}{\partial z}+\nabla \cdot\left(\mathbf{u} s_{i}\right)-\frac{\partial}{\partial z}\left\langle w s_{i}\right\rangle= \\
\left(i^{2}-3 i+1\right) \frac{S_{1} s_{2}+S_{2} s_{1}-s_{3}}{\tau}+v_{i} \nabla^{2} s_{i} .
\end{gathered}
$$

The conservation rule (65) then translates to

$$
\frac{\partial}{\partial z}\left\langle w c_{i}\right\rangle=0, \quad i=1,2 .
$$

where

$$
\left\langle w c_{i}\right\rangle=\left\langle w s_{i}\right\rangle+\left\langle w s_{3}\right\rangle, \quad i=1,2 .
$$

The average of $\widetilde{\mathcal{R}}$ is determined from (54):

$$
\langle\widetilde{\mathcal{R}}\rangle=\frac{1}{\tau}\left\{S_{1} S_{2}-S_{3}+\left\langle s_{1} S_{2}\right\rangle\right\} .
$$

We neglect the third-order terms and, closely following the procedure outlined in section 2, we obtain the nine equations

$$
\begin{gathered}
\frac{\partial S_{i}}{\partial t}+\frac{\partial}{\partial z}\left\langle w s_{i}\right\rangle=\left(i^{2}-3 i+1\right) \frac{S_{1} S_{2}-S_{3}+\left\langle s_{1} s_{2}\right\rangle}{\tau}, \\
i=1,2,3, \\
\frac{\partial}{\partial t}\left\langle w s_{i}\right\rangle+\left\langle w^{2}\right\rangle\left\{\frac{\partial S_{i}}{\partial z}+\frac{\left\langle w s_{i}\right\rangle}{\left\langle w^{2}\right\rangle \tau_{1}}\right\}= \\
\left(i^{2}-3 i+1\right) \frac{S_{2}\left\langle w s_{1}\right\rangle+S_{1}\left\langle w s_{2}\right\rangle-\left\langle w s_{3}\right\rangle}{\tau} \\
+\left(1-b_{1}\right) \frac{g}{T}\left\langle\vartheta s_{i}\right\rangle, \quad i=1,2,3,
\end{gathered}
$$

\footnotetext{
${ }^{\S}$ We are seeking steady-state solutions and let $S_{i}$ be time dependent for computational reasons. However, the sum concentrations $C_{i}$ are assumed constant in time.
} 
and

$$
\begin{aligned}
& \frac{\partial\left\langle\vartheta s_{i}\right\rangle}{\partial t}+\langle w \vartheta\rangle \frac{\partial S_{i}}{\partial z}+\frac{\left\langle\vartheta s_{i}\right\rangle}{\tau_{4}(z)} \\
& \quad=\left(i^{2}-3 i+1\right) \frac{S_{2}\left\langle\vartheta s_{1}\right\rangle+S_{1}\left\langle\vartheta s_{2}\right\rangle-\left\langle\vartheta s_{3}\right\rangle}{\tau} \\
& \quad i=1,2,3
\end{aligned}
$$

where, for computational purposes, the prognostic terms are included.

The nine equations (73), (74) and (75) do not quite constitute a closed system since (73) contains the scalar covariance $\left\langle s_{1} s_{2}\right\rangle$. However, we consider this quantity a small perturbation which can be determined and included later if we initially ignore it. Once we have determined $S_{i}$ in this way, we may derive all second-order scalar-scalar covariances by means of (69). This will be shown in the last part of this section.

Combining the three equations (74) we get, in view of (66) and (71), the two equations

$$
\left\langle w^{2}\right\rangle\left\{\frac{\partial C_{i}}{\partial z}+\frac{\left\langle w c_{i}\right\rangle_{\circ}}{\left\langle w^{2}\right\rangle \tau_{1}}\right\}=\left(1-b_{1}\right) \frac{g}{T}\left\langle\vartheta c_{i}\right\rangle, \quad i=1,2 .
$$

Similarly we obtain from (75)

$$
\langle w \vartheta\rangle \frac{\partial C_{i}}{\partial z}+\frac{\left\langle\vartheta c_{i}\right\rangle}{\tau_{4}(z)}=0, \quad i=1,2 .
$$

We have here left out the prognostic terms and used the fact that $\left\langle w c_{i}\right\rangle=\left\langle w c_{i}\right\rangle_{\circ}$ are constant with height. It is of course no surprise that the last two equations have exactly the same form as the equations (28) and (26) pertaining to conserved scalars.

The equations for the derivation of the scalar-scalar covariances are

$$
\begin{aligned}
\frac{\partial}{\partial t}\left\langle s_{i}^{2}\right\rangle & +2\left\langle w s_{i}\right\rangle \frac{\partial S_{i}}{\partial z} \\
= & 2\left(i^{2}-3 i+1\right) \frac{S_{1}\left\langle s_{i} s_{2}\right\rangle+S_{2}\left\langle s_{i} s_{1}\right\rangle-\left\langle s_{i} s_{3}\right\rangle}{\tau} \\
& -2 v_{s}\left\langle\left(\nabla s_{i}\right)^{2}\right\rangle, \quad i=1,2,3
\end{aligned}
$$

where third-order terms have been ignored. We follow Verver et al. (1997) and use the parameterization of the molecular term

$$
2 v_{s}\left\langle\nabla s_{i} \cdot \nabla s_{j}\right\rangle=\frac{\left\langle s_{i} s_{j}\right\rangle}{\tau_{3}(z)} .
$$

We assume with Verver et al. (1997) that $\tau_{3}$ is equal to $\tau_{4}$. In an equilibrium state (70) then becomes

$$
\begin{array}{r}
\left\langle w s_{i}\right\rangle \frac{\partial S_{i}}{\partial z}=\left(i^{2}-3 i+1\right) \frac{S_{1}\left\langle s_{i} s_{2}\right\rangle+S_{2}\left\langle s_{i} s_{1}\right\rangle-\left\langle s_{i} s_{3}\right\rangle}{\tau} \\
-\frac{\left\langle s_{i}{ }^{2}\right\rangle}{2 \tau_{4}(z)}, \quad i=1,2,3 .
\end{array}
$$

We obtain three more equations from the sumconcentrations $\left(C_{i}, c_{i}\right)=\left(S_{i}+S_{3}, s_{i}+s_{3}\right), i=1,2$ which also follow (69) and (80), with the first term on the righthand side set equal to zero, if we replace $s_{i}$ by $c_{i}$ :

$$
\begin{gathered}
\left\langle w c_{i}\right\rangle \frac{\partial C_{i}}{\partial z}=-\frac{\left\langle c_{i}{ }^{2}\right\rangle}{2 \tau_{4}(z)}, i=1,2, \\
\left\langle w c_{1}\right\rangle \frac{\partial C_{2}}{\partial z}+\left\langle w c_{2}\right\rangle \frac{\partial C_{1}}{\partial z}=-\frac{\left\langle c_{1} c_{2}\right\rangle}{\tau_{4}(z)} .
\end{gathered}
$$

The six equations (80), (81), and (82) can, with patience, great care and with Mathematica (Wolfram, 1999) as a crutch, be solved for $\left\langle s_{1} s_{2}\right\rangle$. First, however, we recast the basic equations (73), (74) and (75) in a dimensionless form as follows

$$
\left\{\begin{array}{c}
\theta \\
f_{i} \\
F_{i} \\
\gamma_{i} \\
\Gamma_{i}
\end{array}\right\}=\left\{\begin{array}{r}
w_{*} t / h \\
\left\langle w s_{i}\right\rangle / w_{*} \\
\left\langle w c_{i}\right\rangle_{\circ} / w_{*} \\
\left\langle\vartheta s_{i}\right\rangle / T_{*} \\
\left\langle\vartheta c_{i}\right\rangle / T_{*}
\end{array}\right\}
$$

and the dimensionless parameter, the Damköhler number,

$$
\mathcal{D}=\frac{h}{w_{*} \tau} .
$$

In dimensionless form (73), (74) and (75) become

$$
\begin{aligned}
\frac{\partial S_{i}}{\partial \theta}+ & \frac{\partial f_{i}}{\partial \zeta}= \\
& \mathcal{D} \times\left(i^{2}-3 i+1\right)\left(S_{1} S_{2}-S_{3}+\left\langle s_{1} S_{2}\right\rangle\right),
\end{aligned}
$$

$$
\begin{aligned}
\frac{\partial f_{i}}{\partial \theta}+\omega^{2}(\zeta)\left\{\frac{\partial S_{i}}{\partial \zeta}+\frac{f_{i}}{\omega^{2} \theta_{1}}\right\}= \\
\\
\qquad \mathcal{D} \times\left(i^{2}-3 i+1\right)\left(S_{2} f_{1}+S_{1} f_{2}-f_{3}\right)+\gamma_{i}
\end{aligned}
$$

and

$$
\begin{aligned}
\frac{\partial \gamma_{i}}{\partial \theta}+\varphi_{T}(\zeta) & \frac{\partial S_{i}}{\partial \zeta}+\frac{\gamma_{i}}{\theta_{4}}= \\
\mathcal{D} & \times\left(i^{2}-3 i+1\right)\left(S_{2} \gamma_{1}+S_{1} \gamma_{2}-\gamma_{3}\right)
\end{aligned}
$$

The summed equations become

$$
\begin{gathered}
\frac{\partial F_{i}}{\partial \zeta}=0, \\
\omega^{2} \frac{\partial C_{i}}{\partial \zeta}-\Gamma_{i}=-\frac{F_{i}}{\theta_{1}}, \quad i=1,2
\end{gathered}
$$

and

$$
\varphi_{T} \frac{\partial C_{i}}{\partial \zeta}+\frac{\Gamma_{i}}{\theta_{4}}=0, \quad i=1,2 .
$$

For $\mathcal{D}=0$, which corresponds to $\tau=\infty$ (i.e. no decay of $\mathrm{NO}_{2}$ ) we obtain the same three equations as (88), (89), 
and (90) in their diagnostic form if we make the replacement $\left(C_{i}, F_{i}, \Gamma_{i}\right) \rightarrow\left(S_{i}, f_{i}, \gamma_{i}\right)$.

Since $F_{i}$ is constant with height we can determine $\partial C_{i} / \partial \zeta$ and $\Gamma_{i}$ as functions of $\zeta$ once $F_{i}$ has been specified. From (89) and (90) we get

$$
\frac{\frac{\partial C_{i}}{\partial \zeta}}{F_{i}}=-\frac{1}{\theta_{1}\left(\omega^{2}+\theta_{4} \varphi_{T}\right)}, \quad i=1,2
$$

and

$$
\frac{\Gamma_{i}}{F_{i}}=\frac{\theta_{4} \varphi_{T}}{\theta_{1}\left(\omega^{2}+\theta_{4} \varphi_{T}\right)}, \quad i=1,2 .
$$

These two equations are equivalent to (36) and (37).

The covariance $\left\langle s_{1} s_{2}\right\rangle$ can now be determined from (80), (81), and (82) in their dimensionless forms:

$$
\begin{gathered}
\frac{f_{i} S_{i}^{\prime}}{\mathcal{D}}=\left(i^{2}-3 i+1\right)\left\{S_{1}\left\langle s_{i} s_{2}\right\rangle+S_{2}\left\langle s_{i} s_{1}\right\rangle-\left\langle s_{i} s_{3}\right\rangle\right\} \\
-q\left\langle s_{i}^{2}\right\rangle, \quad i=1,2,3 \\
\frac{F_{i} C_{i}^{\prime}}{\mathcal{D}}=-q\left\langle c_{i}^{2}\right\rangle=-q\left\{\left\langle s_{i}^{2}\right\rangle+2\left\langle s_{i} s_{3}\right\rangle+\left\langle s_{3}^{2}\right\rangle\right\}, \quad i=1,2,
\end{gathered}
$$

and

$$
\begin{aligned}
\frac{F_{1} C_{2}^{\prime}+F_{2} C_{1}^{\prime}}{2 \mathcal{D}}= & -q\left\langle c_{i}^{2}\right\rangle=-q\left\{\left\langle s_{1} s_{2}\right\rangle+\left\langle s_{2} s_{3}\right\rangle\right. \\
& \left.+\left\langle s_{3} s_{1}\right\rangle+\left\langle s_{3}^{2}\right\rangle\right\}
\end{aligned}
$$

where

$$
\begin{aligned}
S_{i}^{\prime} & =\frac{\partial S_{i}}{\partial \zeta}, \\
C_{i}^{\prime} & =\frac{\partial C_{i}}{\partial \zeta},
\end{aligned}
$$

and

$$
q=\frac{1}{2 \mathcal{D} \theta_{4}} .
$$

We can now construct a set of six linear equations with six unknowns, which are presented in Figure 3 We find that

$$
\left\langle s_{1} s_{2}\right\rangle=-\frac{n u m}{q\left(1+q+S_{1}+S_{2}\right)\left(1+2 q+S_{1}+S_{2}\right)},
$$

where

$$
\begin{aligned}
\text { num } & =A_{11} S_{2}\left(1+S_{1}\right)+A_{22} S_{1}\left(1+S_{2}\right) \\
& -A_{12}\left(1+S_{1}+S_{2}+2 S_{1} S_{2}\right) \\
& +q\left\{\left(A_{11}+A_{22}-2 A_{12}\right)\left(1+S_{1}+S_{2}\right)-A_{12}\right. \\
& \left.-a_{11}\left(1+S_{1}\right)-a_{22}\left(1+S_{2}\right)+a_{33}\right\} \\
& +q^{2}\left\{A_{11}+A_{22}-2 A_{12}-a_{11}-a_{22}\right\} .
\end{aligned}
$$

Here we have used the notation

$$
a_{i i}=\frac{f_{i} S_{i}^{\prime}}{\mathcal{D}}, \quad i=1,2,3,
$$

$$
A_{i i}=\frac{F_{i} C_{i}^{\prime}}{\mathcal{D}}, \quad i=1,2
$$

and

$$
A_{12}=\frac{F_{1} C_{2}^{\prime}+F_{2} C_{1}^{\prime}}{2 \mathcal{D}}
$$

\section{SOLVING THE EQUATIONS}

Here we outline the procedure for solving for concentrations and fluxes, and demonstrate that we can reduce the problem to three equations in $S \equiv S_{3}, f \equiv f_{3}$, and $\gamma \equiv \gamma_{3}$ for the $\mathrm{NO}_{2}$ concentration. ${ }^{\text {II }}$

First we note that we can make the replacements $\left(f_{1}, f_{2}\right)=\left(F_{1}-f_{3}, F_{2}-f_{3}\right)$ and $\left(S_{1}, S_{2}\right)=\left(C_{1}-S_{3}, C_{2}-\right.$ $\left.S_{3}\right)$, where

$$
C_{i}(\zeta)=C_{i}^{\circ}+F_{i} \int_{\zeta}^{\zeta^{\circ}} \frac{\mathrm{d} \zeta^{\prime}}{\theta_{1}\left(\zeta^{\prime}\right)\left(\omega^{2}\left(\zeta^{\prime}\right)+\theta_{4}\left(\zeta^{\prime}\right) \varphi_{T}\left(\zeta^{\prime}\right)\right)}
$$$$
i=1,2, \quad(104)
$$

is obtained by integrating (91). The quantities $C_{i}^{\circ}$ are the sum concentrations at the reference level $\zeta^{\circ}$, which is the top of our model domain. We use $\zeta^{\circ}=1 / 1.2$, which is the level at which the buoyancy flux changes sign. This is also consistent with estimates of the thickness of the entrainment zone (Gryning and Batchvarova, 1994), which is about as far as one can take a parameterized model of this type.

Further, $\gamma$ is eliminated by expressing it in terms of $F_{1}$, $F_{2}$ in the two relations (92) and $f$ and $S$ in the steady-state form of (87) for $\gamma(i=3)$ :

$\gamma(\zeta)=\frac{\theta_{4} \varphi_{T}\left\{\mathcal{D}\left(F_{2} S_{1}+F_{1} S_{2}\right)-\theta_{1}\left(\omega^{2}+\theta_{4} \varphi_{T}\right) \frac{\partial S}{\partial \zeta}\right\}}{\theta_{1}\left\{1+\mathcal{D}\left(1+S_{1}+S_{2}\right) \theta_{4}\right\}\left\{\omega^{2}+\theta_{4} \varphi_{T}\right\}}$.

We have now reduced the problem to solving the two equations (85) and (86) for $i=3$ :

$$
\frac{\partial S}{\partial \theta}+\frac{\partial f}{\partial \zeta}=\mathcal{D}\left(S_{1} S_{2}-S+\left\langle s_{1} s_{2}\right\rangle\right)
$$

$$
\frac{\partial f}{\partial \theta}+\omega^{2}\left\{\frac{\partial S}{\partial \zeta}+\frac{f}{\omega^{2} \theta_{1}}\right\}=\mathcal{D}\left(S_{2} f_{1}+S_{1} f_{2}-f\right)+\gamma .
$$

These two equations are solved for $S$ and $f$ by iteration under the assumption that the covariance $\left\langle s_{1} s_{2}\right\rangle$ can be considered a perturbation which is neglected in the first calculation and then, determined by (99), inserted in the second calculation.

We are seeking a steady-state solution for the concentrations and fluxes. This is obtained by solving (106)

\footnotetext{
II We have omitted the subscript 3 for convenience.
} 


$$
\left\{\begin{array}{rrrrrr}
-\left(S_{2}+q\right) & 0 & 0 & -S_{1} & 0 & 1 \\
0 & -\left(S_{1}+q\right) & 0 & -S_{2} & 1 & 0 \\
0 & 0 & -(1+q) & 0 & S_{1} & S_{2} \\
-q & 0 & -q & 0 & 0 & -2 q \\
0 & -q & -q & 0 & -2 q & 0 \\
0 & 0 & -q & -q & -q & -q
\end{array}\right\} \times\left\{\begin{array}{c}
\left\langle s_{1}^{2}\right\rangle \\
\left\langle s_{2}^{2}\right\rangle \\
\left\langle s_{3}^{2}\right\rangle \\
\left\langle s_{1} s_{2}\right\rangle \\
\left\langle s_{2} s_{3}\right\rangle \\
\left\langle s_{3} s_{1}\right\rangle
\end{array}\right\}=\left\{\begin{array}{c}
\frac{f_{1} S_{1}^{\prime}}{\mathcal{D}} \\
\frac{f_{2} S_{2}^{\prime}}{\mathcal{D}} \\
\frac{f_{3} S_{3}^{\prime}}{\mathcal{D}} \\
\frac{F_{1} C_{1}^{\prime}}{\mathcal{D}} \\
\frac{F_{2} C_{2}^{\prime}}{\mathcal{D}} \\
\frac{F_{1} C_{2}^{\prime}+F_{1} C_{1}^{\prime}}{2 \mathcal{D}}
\end{array}\right\}
$$

Figure 3: The matrix formulation of the six linear equations.

and (107) as partial differential equations with the time $\theta$ and the height $\zeta$ as independent variables, using a relaxation scheme in $\theta$. The boundary conditions for these steady-state solutions are the surface fluxes $f_{1}, f_{2}$, and $f=f_{3}$ and three flux-concentration relations at the top of the boundary layer. The first three are applied to obtain $F_{1}$ and $F_{2}$ in (104). The next three are specifications of fluxes at the top of the boundary layer in terms of a dimensionless entrainment velocity $\omega_{e}=w_{e} / w_{*}{ }^{\|}$and the differences between the concentrations in the free troposphere and those at a reference level $\zeta^{\circ}$ inside the boundary layer. To proceed we need expression for $w_{e}$. The entrainment process at the top of the CBL is independent of the species, and, if we take the potential temperature as a marker, we have

$$
\langle\vartheta w\rangle_{h}=-w_{e} \Delta \Theta,
$$

where $\Delta \Theta$ is the jump in potential temperature across the top $h$ of the CBL. In (40) we have already assumed that $\langle\vartheta w\rangle_{h}=-0.2\langle\vartheta w\rangle_{\circ}$ so that

$$
w_{e}=0.2 \frac{\langle\vartheta w\rangle_{\circ}}{\Delta \Theta} .
$$

The magnitude of the the jump has been estimated by Tennekes (1973) who expressed it in terms of the gradient $\Gamma_{\Theta}$ of the potential temperature in the free troposphere and $h$ by

$$
\Delta \Theta=\frac{1}{7} \Gamma_{\Theta} h .
$$

Inserting this result into (101), we get

$$
w_{e}=1.4 \frac{\langle w \vartheta\rangle_{\circ}}{\Gamma_{\Theta} h}=\frac{1.4}{1-b_{1}} w_{*}\left(\frac{\frac{g}{T} \Gamma_{\Theta}}{\frac{w_{*}^{2}}{h^{2}}}\right)^{-1},
$$

\footnotetext{
$\|$ By convention the entrainment velocity is positive when the flux is downward.
}

where the fraction inside the parenthesis can be identified as a Richardson number based on the potentialtemperature gradient in the free troposphere and freeconvection turbulence in a CBL of depth $h$. With the typical values $\Gamma_{\Theta}=10^{-2} \mathrm{~K} \mathrm{~m}^{-1}, h=10^{3} \mathrm{~m}$, and $w_{*}=1 \mathrm{~m}$ $\mathrm{s}^{-1}$, we find that $\omega_{e}=7 \times 10^{-3}$.

Denoting concentrations in the free atmosphere by an asterisk superscript, we have:

$$
\left\{\begin{array}{l}
f_{1}^{\circ} \\
f_{2}^{\circ} \\
f^{\circ}
\end{array}\right\}=\omega_{e} \times\left\{\begin{array}{ll}
S_{1}^{\circ}-S_{1}^{*} & \\
S_{2}^{\circ}-S_{2}^{*} & \\
S^{\circ}-S^{*}, & (i=3)
\end{array}\right\} .
$$

We may reduce the degrees of freedom by assuming photostationary equilibrium Leighton (1961) between the three species:

$$
k \tau \frac{[\mathrm{NO}]^{*}\left[\mathrm{O}_{3}\right]^{*}}{\left[\mathrm{NO}_{2}\right]^{*}}=\frac{S_{1}^{*} S_{2}^{*}}{S^{*}}=1 .
$$

We note that, the way the problem is now presented, it is symmetric in the indices 1 and 2. This means that we may always assume that $S_{1}^{\circ} \geq S_{2}^{\circ}$ at the reference level $\zeta^{\circ}$ and in the free troposphere.

\section{SAMPLE CALCULATION}

Here we show a set of plots from a sample run of the model. The lower limit for the spatial integration cannot be zero because concentrations are not finite at $z=0$. Therefore we have chosen the lower limit to be $\zeta_{\circ}=10^{-5}$, which is consistent with surface layer formulations which are considered to be valid only down to a surface roughness length that is determined by the properties of the surface. As noted earlier, the top of the domain is at $\zeta^{\circ}=1 / 1.2$. 
In figures $4,5,6$, and 7 we use $\omega_{e}=0.07$ and $\mathcal{D}=4$, and the boundary conditions $S_{1}^{*}=1, S_{2}^{*}=$ $0.01, f_{1}\left(\zeta_{0}\right)=-0.0036, f_{2}\left(\zeta_{0}\right)=0.0049$, and $f_{3}\left(\zeta_{0}\right) \equiv$ $f\left(\zeta_{0}\right)=-0.0001$. In the first two figures, the covariance term $\left\langle s_{1} s_{2}\right\rangle$ is set equal to zero, while in the second two figures the term is included. We see that there is a small, but significant, difference between the two sets of curves.

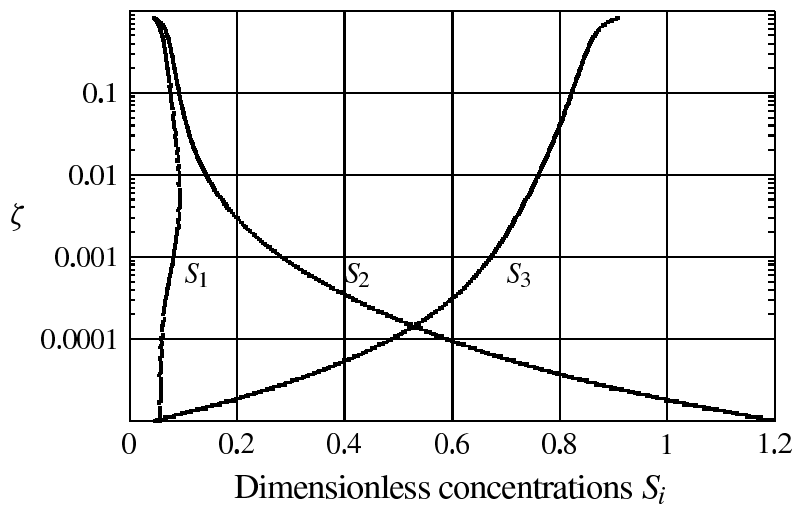

Figure 4: Concentration profiles with $\left\langle s_{1} s_{2}\right\rangle=0$.

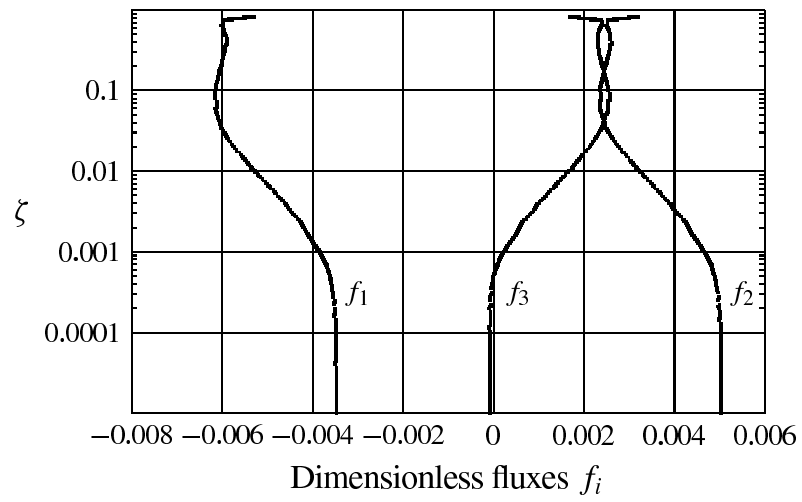

Figure 5: Flux profiles with $\left\langle s_{1} s_{2}\right\rangle=0$.

\section{Acknowledgements}

We thank John Orlando, Thomas Karl and Alex Guenther for their help in trying to educate the chemically challenged authors of this manuscript.

\section{REFERENCES}

André, J. C., G. D. Moor, , P. Lacarrère, and R. D. Vachat, 1976: Turbulent approximation for inhomogeneous flows: Part 2. the numerical simulation of a penetrative convection experiment, J. Atmos. Sci., 33, 482-491.

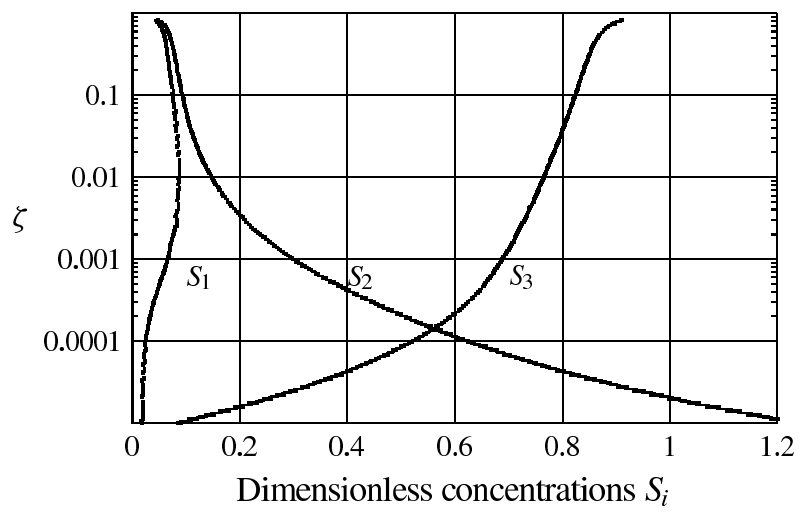

Figure 6: Concentration profiles with $\left\langle s_{1} s_{2}\right\rangle$ included.

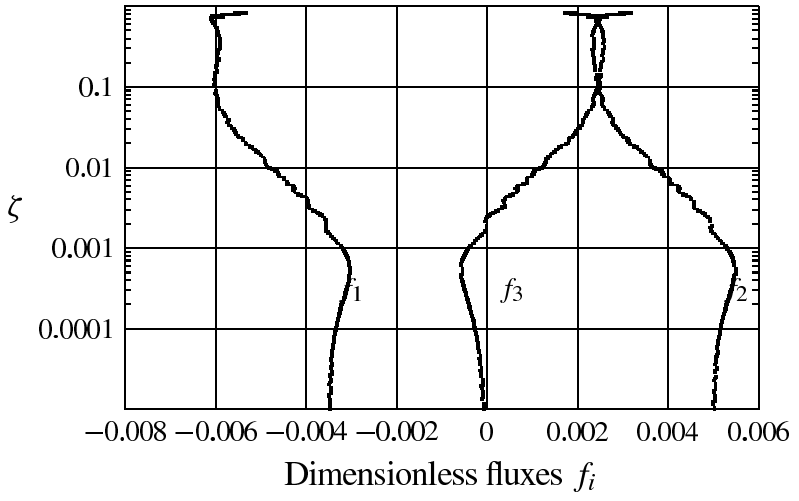

Figure 7: Flux profiles with $\left\langle s_{1} s_{2}\right\rangle$ included.

Busch, N. E., 1973: On the mechanics of atmospheric turbulence, in Workshop on Micrometeorology, edited by D. A. Haugen, pp. 1-28, American Meteorological Society.

Gryning, S.-E. and E. Batchvarova, 1994: Parameterization of the depth of the entrainment zone above the daytime mixed layer, Quart. J. Roy. Meteorol. Soc., 120, 47-58.

Holtslag, A. A. M. and C. Moeng, 1991: Eddy diffusivity and countergradient transport in the convective atmospheric boundary layer, J. Atmos. Sci., 48, 1690-1698.

Kristensen, L., C. E. Andersen, H. E. Jørgensen, P. Kirkegaard, and K. Pilegaard, 1997: First-order chemistry in the surface-flux layer, J. Atmos. Chem., 27, 249-269.

Kristensen, L. and P. Kirkegaard, 2006: Fluxes and concentrations of non-conserved scalars in the atmospheric surface layer, second-order destruction, J. Atmos. Chem., 53, 251-283.

Leighton, P. A., 1961: Photochemistry of Air Pollution, Academic Press, New York, 300 pp. 
Lenschow, D. H. and A. C. Delany, 1987: An analytic formulation of $\mathrm{NO}$ and $\mathrm{NO}_{2}$ flux profiles in the atmospheric surface layer, J. Atmos. Chem., 5, 301-309.

Lenschow, D. H., J. C. Wyngaard, and W. T. Pennell, 1980: Mean-field and second-moment budgets in a baroclinic, convective boundary layer, J. Atmos. Sci., 37, 1314-1326.

Moeng, C. and J. C. Wyngaard, 1989: Evaluation of turbulent transport and dissipation closures in secondorder modelling, J. Atmos. Sci., 46, 2311-2330.

Ridley, B. A., S. Madronich, R. B. Chatfield, J. G. Walega, R. E. Shetter, M. A. Carrol, and D. D. Montzka, 1992: Measurements and model simulations of the photostationary state during the Mauna-Loa-observatory photochemistry experiment-implications for radical concentrations and ozone production and loss rates, J. Geophys. Res.Atm., 97 (D10), 10375-10388.

Tennekes, H., 1973: A model for the dynamics of the inversion above a convective boundary layer, J. Atmos. Sci., 30, 558-567.

Verver, G. H. L., H. van Dop, and A. A. M. Holtslag, 1997: Turbulent mixing of reactive gases in the convective boundary layer, Boundary-Layer Meteorol., 85, 197-222.

—, 2000: Turbulent mixing and the chemical breakdown of isoprene in the atmospheric boundary layer, J. Geophys. Res., 105, 3983-4002.

Wolfram, S., 1999: The Mathematica Book, 4th ed., Wolfram Media/Cambridge University Press, 1470 pp.

Wyngaard, J. C., 1982: Boundary-layer modeling, in Atmospheric Turbulence and Air Pollution Modeling, edited by F. T. M. Nieuwstadt and H. van Dop, chapter 3, pp. 69-106, D. Reidel Publishing Company, Dordrecht, Holland, Boston, U.S.A., London, England.

Wyngaard, J. C. and R. A. Brost, 1984: Top-down and bottom-up diffusion of a scalar in the convective boundary layer, J. Atmos. Sci., 41, 102-112. 